ISSN: 2639-0531

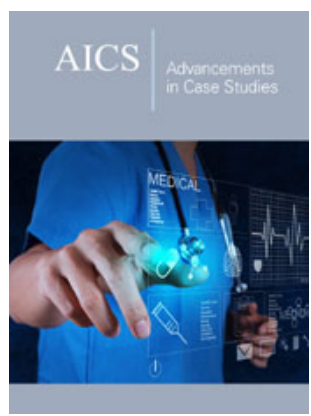

*Corresponding author: Lei Huang, The 302 Hospital of PLA, Treatment and Research Center for Infectious Diseases, China

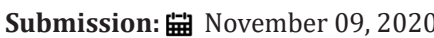

Published: 垱 November 20, 2020

Volume 2 - Issue 5

How to cite this article: Xiaochun $\mathrm{Li}$, Wenwen He, Yifang Wen, Wei Jiang, Lei Huang*. The Role of Blood Microbiome and Microbial Product Translocation in Disease Pathogenesis. Adv Case Stud. 2(5). AICS.000548. 2020.

DOI: 10.31031/AICS.2020.02.000548

Copyright@ Lei Huang, This article is distributed under the terms of the Creative Commons Attribution 4.0 International License, which permits unrestricted use and redistribution provided that the original author and source are credited.

\section{The Role of Blood Microbiome and Microbial Product Translocation in Disease Pathogenesis}

Xiaochun $\mathrm{Li}^{1}$, Wenwen $\mathrm{He}^{2}$, Yifang Wen ${ }^{3}$, Wei Jiang ${ }^{4,5}$ and Lei Huang ${ }^{6 *}$

${ }^{1}$ The Sixth People's Hospital of Xinjiang, Antiviral Treatment Center for HIV/AIDS, China

${ }^{2}$ The Sixth People's Hospital of Xinjiang, Clinical Laboratory Center, the Sixth People's hospital, China

${ }^{3}$ The Sixth People's Hospital of Xinjiang, Hepatitis Treatment Center, China

${ }^{4}$ Department of Microbiology and Immunology, Medical University of South Carolina, USA

${ }^{5}$ Divison of Infectious Diseases, Department of Medicine, USA

${ }^{6}$ The 302 Hospital of PLA, Treatment and Research Center for Infectious Diseases, China

\begin{abstract}
Circulation has been considered in a sterile condition in individuals without live microbial infections. However, recent publications from our group and others highlight detection of blood microbiome or microbial molecules in healthy individuals and individuals with various diseases, as well as their potential roles in disease pathogenesis. In this review, we introduce the published studies and research advancement in the field of blood microbial and microbial translocation in disease immunopathogenesis.
\end{abstract}

Keywords: Blood microbiome; Microbial product translocation; Disease pathogenesis

\section{Introduction}

Circulation has been considered in a sterile condition in individuals without live microbial infections. However, recent publications highlight detection of blood microbiome or microbial molecules in healthy individuals and individuals with various diseases without a live infection or sepsis. Due to the low blood levels of microbial biomass and background contaminations from experimental procedures, blood microbiome analysis has been met with technical challenges. Nonetheless, blood microbiome provides important information to study the interactions of microbiome and hosts, which is potentially critical for investigating their effects on host immune perturbations in various disease conditions.

Blood and tissue microbiome have been studied in some fields. In a recent study, blood and tissue microbiome has been assessed and cancer-specific microbial sequences have been identified in different types of cancer in humans [1]. In another study with seven different type of cancer, evidence was found that cancer-specific bacteria were detected within both immune cells and cancer tissues [2].

We have extensively investigated both quantity and quality of blood microbial translocation and their roles in HIV pathogenesis. In HIV, the magnitude of blood microbial translocation correlates with $\mathrm{T}$ cell activation, B cell activation and apoptosis, autoantibody production, persistent immune activation and inflammation, immune recovery after antiretroviral therapy, and organ complications [3-9]. Notably, not only the total amount of microbial translocation, but also the specific bacterium or its molecule translocation plays a role in HIV disease pathogenesis. In a recent study, we found that translocation of Staphylococcus aureus plays a role in autoantibody production in HIV, and provide evidence of systemic exposure of Staphylococcus aureus drove germinal central autoreactive B cell activation and autoantibody production in mice [8].

In autoimmune diseases, studies from Silverman's group and Kriegel's group reveal that translocation of gut pathobionts drive autoimmune diseases [10-13]. Our recent 
studies in systemic lupus erythematosus (SLE) disease show that plasma microbial dysbiosis in the first-degree relatives of lupus patients compared to unrelated healthy controls, and plasma levels of microbial product translocation correlated with levels of autoantibodies in lupus patients and first-degree relatives of patients $[14,15]$.

Studies in humans with chronic inflammatory diseases show that blood microbiome was detected and suggest that blood microbiome may play a role in the production of inflammation [16-18]. Moreover, blood and adipose tissue microbiome has been identified and suggest its role in tissue inflammation in obesity and type 2 diabetes; gut microbial translocation to the pancreatic lymph nodes triggered NOD2 singling pathway and contributed to type 1 diabetes $[19,20]$. Blood microbiome and microbial translocation have been studied in central nervous system diseases as well. An oral bacterium, Porphyromonas gingivalis, has detected to translocate to the brain and plays a role in Alzheimer's disease [21]. Moreover, bacterial LPS has shown to play a role in neurodegenerative diseases [22-24].

\section{Conclusion}

In summary, solid evidence has shown the detection of blood microbiome and microbial product translocation in humans without live infection. Further studies need to explore the mechanisms and causality using animals as well as in humans with treatment against specific microbes.

\section{Acknowledgement}

This work was supported by the National Natural Science Foundation of China (81772185, Huang).

\section{References}

1. Poore GD, Kopylova E, Zhu Q Carpenter C, Fraraccio S, et al. (2020) Microbiome analyses of blood and tissues suggest cancer diagnostic approach. Nature 579(7800): 567-574.

2. Nejman D, Livyatan I, Fuks G, Gavert N, Zwang Y, et al. (2020) The human tumor microbiome is composed of tumor type-specific intracellular bacteria. Science 368(6494): 973-980.

3. Jiang W, Younes SA, Funderburg NT, Mudd JC, Espinosa E, et al. (2014) Cycling memory CD4 + T cells in HIV disease have a diverse T cell receptor repertoire and a phenotype consistent with bystander activation. J Virol 88(10): 5369-5380.

4. Zhang L, Luo Z, Sieg SF, Funderburg NT, Yu X, et al. (2014) Plasmacytoid dendritic cells mediate synergistic effects of HIV and lipopolysaccharide on CD27+ IgD- memory B cell apoptosis. J Virol 88(19): 11430-11441.

5. Luo Z, Ma L, Zhang L, Martin L, Wan Z, et al. (2016) Key differences in $B$ cell activation patterns and immune correlates among treated HIVinfected patients versus healthy controls following influenza vaccination. Vaccine 34(16): 1945-1955.

6. Xu W, Luo Z, Alekseyenko AV, Martin L, Wan Z, et al. (2018) Distinct systemic microbiome and microbial translocation are associated with plasma level of anti-CD4 autoantibody in HIV infection. Sci Rep 8(1): 12863.

7. Zhou Z, Powell AM, Ramakrishnan V, Eckard A, Wagner C, et al. (2018) Elevated systemic microbial translocation in pregnant HIV-infected women compared to HIV-uninfected women, and its inverse correlations with plasma progesterone levels. J Reprod Immunol 127: 16-18.

8. Luo Z, Li M, Wu Y, Meng Z, Martin L, et al. (2019) Systemic translocation of Staphylococcus drives autoantibody production in HIV disease. Microbiome 7(1): 25.

9. Huang L, Deng J, Lang R, Liao G, Jiang W (2020) Enriched LPS staining within the germinal center of a lymph node from an HIV-infected longterm non-progressor but not from progressors. J Immunol Res 2020: 7471380 .

10. Manfredo Vieira S, Hiltensperger M, Kumar V, Zegarra Ruiz D, Dehner C, et al. (2018) Translocation of a gut pathobiont drives autoimmunity in mice and humans. Science 359(6380): 1156-1161.

11. Azzouz D, Omarbekova A, Heguy A, Schwudke D, Gisch N, et al. (2019) Lupus nephritis is linked to disease-activity associated expansions and immunity to a gut commensal. Annals of the Rheumatic Diseases 78(7): 947-956.

12. Ruff WE, Dehner C, Kim WJ, Pagovich O, Aguiar CL, et al. (2019) Pathogenic autoreactive $\mathrm{T}$ and $\mathrm{B}$ cells cross-react with mimotopes expressed by a common human gut commensal to trigger autoimmunity. Cell Host Microbe 26(1): 100-113.

13. Silverman GJ (2019) The microbiome in SLE pathogenesis. Nat Rev Rheumatol 15(2): 72-74.

14. Ogunrinde E, Zhou Z, Luo Z, Alekseyenko A, Li QZ, et al. (2019) A link between plasma microbial translocation, microbiome, and autoantibody development in first-degree relatives of systemic lupus erythematosus patients. Arthritis Rheumatology 71(11): 1858-1868.

15. Ogunrinde E, Zhou Z, Gilkeson G, Jiang W (2019) The limitation and racial difference of the plasma microbiome in SLE disease. Arthritis Rheumatology 71(12): 2127.

16. Potgieter M, Bester J, Kell DB, Pretorius E (2015) The dormant blood microbiome in chronic, inflammatory diseases. FEMS Microbiology Reviews 39(4): 567-591.

17. Lelouvier B, Servant F, Paisse S, Brunet AC, Benyahya S, et al. (2016) Changes in blood microbiota profiles associated with liver fibrosis in obese patients: A pilot analysis. Hepatology 64(6): 2015-2027.

18. Luo Z, Li M, Wu Y, Meng Z, Martin L, et al. (2019) Systemic translocation of Staphylococcus drives autoantibody production in HIV disease. Microbiome 7(1): 25.

19. Massier L, Chakaroun R, Tabei S, Crane A, Didt KD, et al. (2020) Adipose tissue derived bacteria are associated with inflammation in obesity and type 2 diabetes. Gut 69(10): 1796-1806.

20. Costa FR, Francozo MC, De Oliveira GG, Ignacio A, Castoldi A, et al. (2016) Gut microbiota translocation to the pancreatic lymph nodes triggers NOD2 activation and contributes to T1D onset. J Exp Med 213(7): 12231239.

21. Dominy SS, Lynch C, Ermini F, Benedyk M, Marczyk A, et al (2019) Porphyromonas gingivalis in Alzheimer's disease brains: Evidence for disease causation and treatment with small-molecule inhibitors. Sci Adv 5(1): eaau3333.

22. Martins IJ (2015) Overnutrition determines LPS regulation of mycotoxin induced neurotoxicity in neurodegenerative diseases. Int J Mol Sci 16(12): 29554-29573.

23. IJ M (2017) Antibiotic resistance involves antimicrobial inactivation in global communities. Scholarena Journal of Pharmacy and Pharmacology 1(1): 1-2.

24. Martins I (2018) Bacterial lipopolysaccharides and neuron toxicity in neurodegenerative diseases. Neurology Research \& Surgery 1(1): 1-3.

For possible submissions Click below: 